\title{
Procalcitonin: Blood tests are useful but must be interpreted with caution - inflammatory markers are no exception
}

An article in this edition deals with the worthy task of assessment of an inflammatory marker, procalcitonin (PCT), to identify pregnancyassociated sepsis (PAS) predominantly due to bacterial infection. It addresses the need to establish normal values, deviation from which might alert the clinician to infection, or which may guide the clinician in assessing the path to recovery or the requirement for further intervention should response to treatment seem, according to assessment of PCT, to be poor. ${ }^{[1]}$

The numbers in the study are relatively small, and the authors acknowledge that with even greater division into the three trimesters and beyond, confident establishment of the range of normal values is not possible. This is a pilot study. Larger studies exist, and several are quoted by this article.

The authors acknowledge that a recognised application of PCT is serial measurement, and this was not evaluated. A true knowledge of how PCT performs over time may be almost as important in avoiding misinterpretation. An analogy could be the specific patterns of beta human chorionic gonadotrophin (hCG) change over time that were once thought typical of ectopic pregnancy - it is now realised that almost any pattern of beta hCG (rising, static or falling) may be associated with ectopic pregnancy. ${ }^{[2]}$ This is to highlight the fact that limited understanding of a blood parameter may lead to underdiagnosis or misunderstanding of a process.

Regarding PCT, underdiagnosis or overdiagnosis of sepsis, or overdiagnosis of a poor response to treatment, might occur by incorrect interpretation of PCT values or changes over time.

PCT is one marker of inflammation or sepsis. Others include the white cell count (WCC), and C-reactive protein (CRP). Erythrocyte sedimentation rate (ESR) is also used. A falling platelet count or thrombocythaemia may also point to sepsis. Aspartate transaminase, which is more normally, in obstetrics, associated with the liver damage associated with pre-eclampsia, may also act as an inflammatory marker or acute-phase reactant.

This introduces the issue of confounding variables into the topic of laboratory assessment - in the case of PCT, this means that other conditions unrelated to acute infection may mislead, or may suggest persistent infection when, for example, the patient is recovering.

We are all familiar with false positives that incorrectly point to infection: the mainstay test for syphilis in pregnancy, the rapid plasma reagin test (RPR), may suggest syphilitic infection, but may instead be identifying an auto-immune condition or another false positive. Some clinicians may not know the extent of reported misdiagnosis with RPR - in one series, a false positive was reported in as many as $60 \%$ of cases. ${ }^{[3]}$ Such false positives can have serious and unjustified consequences.

For PCT, the authors highlight several conditions that may produce false positive results for PAS - pre-eclampsia, premature rupture of membranes, trauma, the rarer liver cirrhosis and malignancies of the thyroid or lung. PCT has also, however, been shown to be raised in paralytic ileus without confirmed intra-abdominal infection, ${ }^{[4]}$ though the two may coincide.
A false positive PCT might lead to the expense of an extended hospital stay or a prolonged stay in a high care/intensive care setting with an increased risk of hospital-acquired infection, and where increased scrutiny may be beneficial or may lead to inappropriate intervention. A false positive may lead to a change to more expensive or less easily accessed antibiotics.

Were a false positive elevated PCT interpreted as an unresolving abdominal infection, particularly at the urgent and dangerous time in the days after caesarean delivery, this could lead to inappropriate re-look laparotomy, or even inappropriate hysterectomy, in a setting where severe sepsis is more often seen.

As suggested in the theoretical example above, even when a raised PCT is genuinely identifying a focus of bacterial sepsis, as with any marker, it does not show where that focus may be. Patients who are ventilated over a period of time may easily develop respiratory infections; if there is limited or a delayed response to antibiotics, a persistently raised or slowly falling PCT may encourage attention to be directed to the abdomen, where infection may not be present, and so, once again, unnecessary re-look laparotomy may be performed a procedure sometimes seemingly considered with the ease of a full blood count.

In such a situation, supportive tests to confirm a source of sepsis, such as ultrasound of the abdomen, may be prone to false positives or false interpretation also. Fluid seen on imaging in the abdomen postoperatively may lead to a suspicion of sepsis. But the presence of free serous fluid inside the abdomen may be a natural phenomenon in the previously unwell postoperative patient who has comorbid conditions that may include a lowered albumin, causing oncotic pressure to fall. ${ }^{[5]}$ Ultrasound or computed tomography may give some indication of the character of the fluid. But it may not. The absence of a falling haemoglobin $(\mathrm{Hb})$ may show that the fluid is not blood (though some may fail to understand that the intra-assay variability for an $\mathrm{Hb}$, the range of results seen in repeated tests on a single sample, can be as high as $10 \%$ or a little more, ${ }^{[6]}$ and may falsely interpret a modest fall). In the presence of a raised PCT or similar inflammatory marker (also subject to intra-assay variability), fluid seen on imaging may lead to an intra-abdominal search for pus that is not present in a recovering patient.

How does the physician avoid unnecessary conclusions from raised levels of inflammatory markers, or from a failure to rise, and so miss sepsis, or be liable to misdirection in the search for sepsis? A proper history for appropriate symptoms and a thorough physical examination are essential; this will be limited in an intubated patient - but still an accurate search for physical signs is essential.

In the awake patient the identification of a medical problem or its severity by clinical patient assessment is too frequently ignored in favour of a preference for laboratory parameters and complex imaging machines, although these may be helpful, or even very helpful.

Inflammatory markers such as PCT may assist greatly in the care of an unwell patient. A thorough knowledge of normal values and trends over time is essential. But the patient should still be observed. 
A patient who moves easily in bed with a resolving tachycardia and minimal abdominal tenderness and who smiles in response to questions does not obviously need a re-look laparotomy.

Establishing normal values in pregnancy, and at different times in pregnancy and in different conditions, is vital. There are still some clinicians who are not aware that a blood WCC of up to $15 \times 10^{9}$ may be normal in pregnancy, and that values of $20 \times 10^{9}$ may normally occur in the puerperium. ${ }^{[7]}$ Many errors may have been caused by misinterpretation of these values, just as a physician may be misled by an S1Q3T3 electrocardiograph pattern and a positive D-dimer in pregnancy, incorrectly diagnosing a pulmonary embolus in a patient sitting in bed, without oxygen, conversing easily, with a clear history of indigestion or reflux and a pulse rate of 80 beats per minute who is, therefore, less likely to have cardiovascular or respiratory compromise associated with an embolus.

Cost should also be an important driver of medical care: where infection is obvious, confirmation by PCT may be costly and unnecessary, and the path to recovery may be as easily charted by a falling temperature and pulse, and by improvement in the patient's condition.

\section{William Edridge}

Editor

william.edridge@gmail.com

S Afr J Obs Gynae 2019;25(1):2-3. DOI:10.7196/SAJOG.2019.v25i1.1484

1. Agarwal R, Priyadarshini P, Mehndiratta M. Serum procalcitonin in pregnancy-associated sepsis A case control study. S Afr J Obs Gynae 2019;25(1):15-19. https://doi.org/10.7196/SAJOG.2019. v25il.1397

2. Surampudi K, Gundabattula SR. The role of serum beta hCG in early diagnosis and management strategy of ectopic pregnancy. J Clin Diagn Res 2016;10(7):QC08-QC10. https://doi.org/10.7860/ JCDR/2016/19342.8110

3. Arora S, Doda V, Rani S, Kotwal U. Rapid plasma reagin test: High false positivity or important marker of high risk behaviour. Asian J Transfus Sci 2015;(1):109. https://doi.org/10.4103/0973 6247.150979

4. Secmeer G, Devrim I, Kara A, et al. The role of procalcitonin and CRP in differentiating a stable from a deteriorating clinical course in paediatric febrile neutropenia. J Pediatr Hematol Oncol 2007;29(2):107-111.

5. Mayberry JC, Welker KJ, Goldman RK, Mullins RJ. Mechanism of acute ascites formation after trauma resuscitation. Arch Surg 2003;138(7):773-776. https://doi.org/10.1001/archsurg.138.7.773

6. Parker M, Han Z, Abu-Haydar E, et al. An evaluation of haemoglobin measurement tools and their accuracy and reliability when screening for child anaemia in Rwanda: A randomised study. PLOS ONE 2018;13(1):e0187663. https://doi.org/10.1371/journal.pone.0187663

7. Chandra S, Tripathi AK, Mishra S, Amzarul M, Vaish AK. Physiological changes in haematological parameters during pregnancy. Indian J Hematol Blood Transfus 2012;28(3):144-146. https://doi. org/10.1007/s12288-012-0175-6 